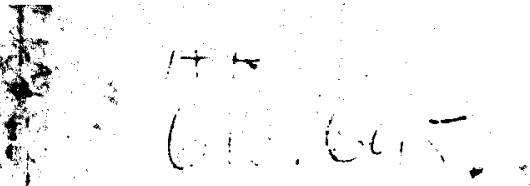

BANAGNMFNR SCIFACES FOR HWALTI

A No:s PoYtT Nastrttov

The Village Health Wo-ker:

The Need and Potential as Determined bv Survey in Three Provinces of Afghanistan

January, 1977

$\quad$ Prepared uy: Kevin i. Deniny
Timothy N. Warrer

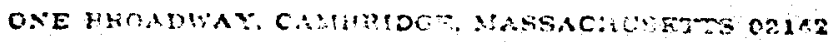

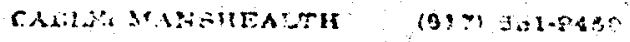
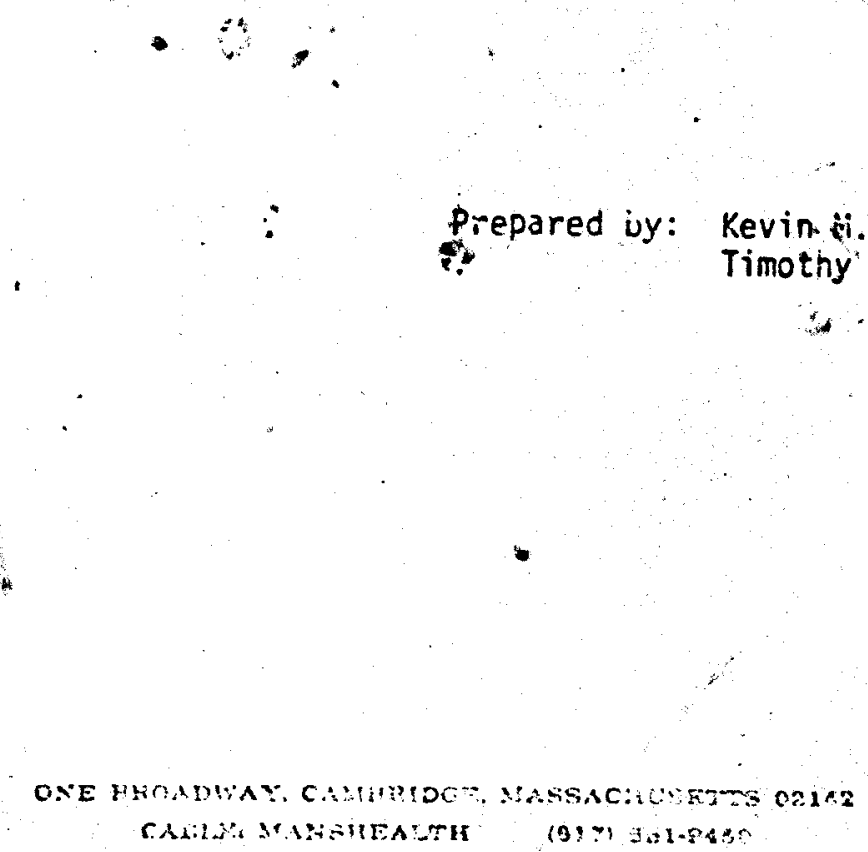
Table of Contents

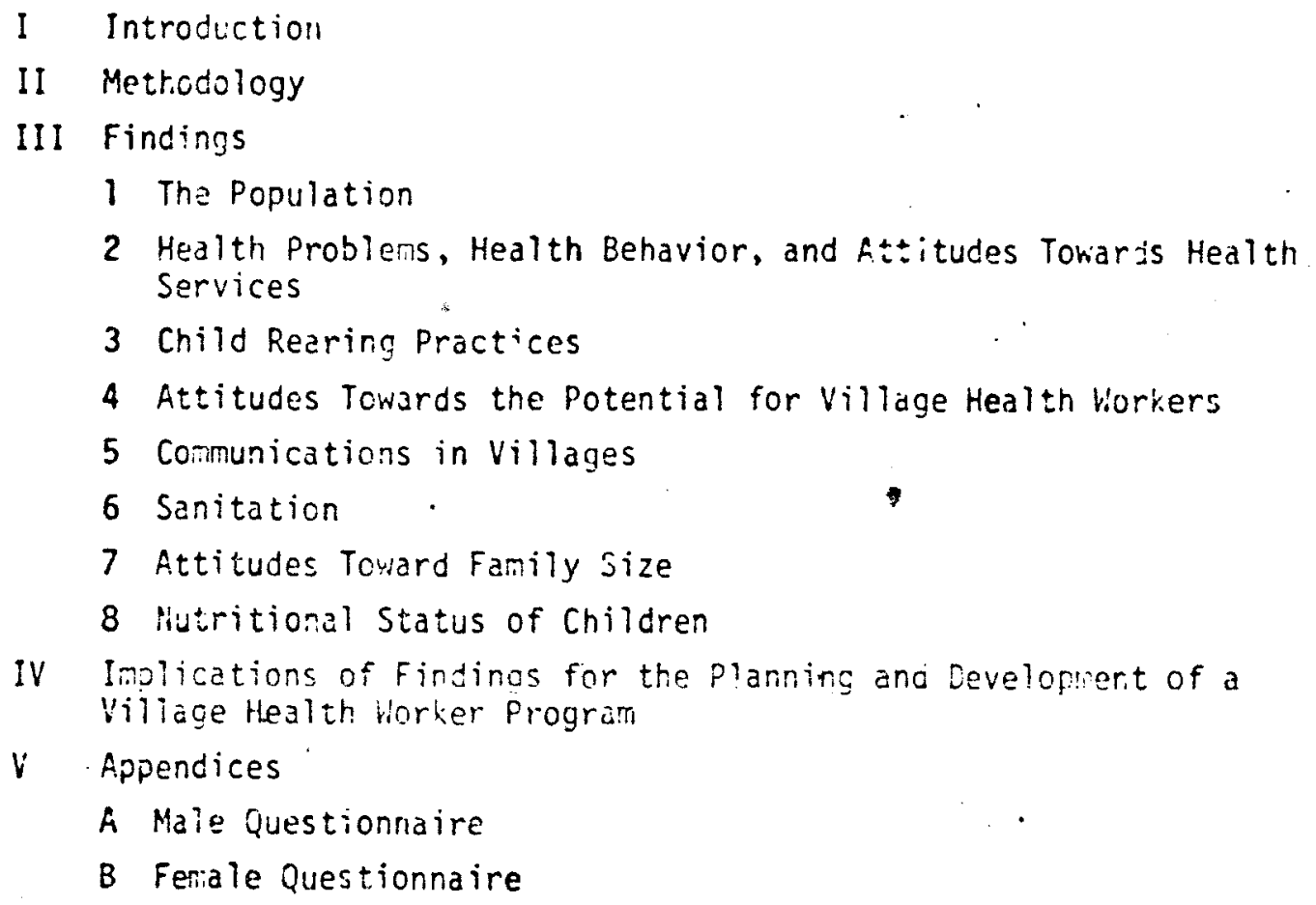

? 
I. Introdiction

The study of health practices, attitudes and neess in three provinces of Afgharistun, results of which are presented in this prelimirary report, had several objectives. The primary ones were co otiain additional information on the means by which villagers meet their health needs and to collect information to be used in planning local healih services, especially the development of a viliage health worker scheme.

The study, which was carried out from August to "lovelnber, 1976, complenents and augments"a prior study, "A Field Survey of Health Necds, Practices and Resources in Rural Afghanistan", which was conducted at five basic health centers in the Parwan-Kapisa Province in the sumer of 1974. While much of the information nbtained in, the Parwan Survey parallels that of the nore recent Three Province Study and can be used comparativeiy, the population of this subsequent survey has been selected to be more reuresentative of Afghanistan as a nation than was that scudied in the Parvan-Kapisa survey. Not unly are the urovinces selected - Ghazni, Bughian and Helmand--gecgraphicaliy, ethrically and economically different from eech other, but the sites within each province ave also been selected to represent the variety in village structure and location. For instance, in each province, villages have been selected a $i$ varying distances from the expstiat Sasic health Center in crder to allow for comparison in health patterris and uses of BHC's services by access. Another difference between studies is that in the Three Provinca Survey, all interviews were conductio in households systematically selected from within villages: interviewing in the prior survey was restricted to the vicinity of the basic health center.

A major addition in the Three Province Survey has becn a set of questions to elicit information as to villagers' attitudes towards the best means by which their own needs can be met. Specifically, respondents were asked to report their feelings as to the feasitility of a villace realth worker sctane for their own viliage and the raans by utirh such workers could best to selected and trainej. Thay were diso questiunid 


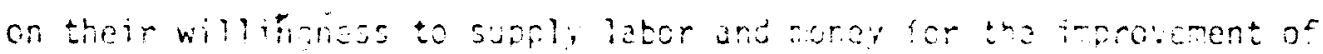
villoge entalica and water supply.

4s in the previous survey, emphasis has boen pleced on obtaining information on child nutritional practices, an area of utmost inter ast in the planning of iocal level heaith and educat onsi orograms. Heignts, heights and upper arm circum.erences were cbtained for children under five years of age to be used as an estimate of the nutritional status of rural Afghan children.

Finally, in this report, some of the imp'ications of preliminary findings for the planning and management of a village Health korker program for rural Afghanistan will be discussed.

This preliminary repart will be followed by a more detailed comprehensive report wich will explore in groater delth the complex interactions between health problems and health actions in the severiteen villages stusied. 
II. Metrodiga

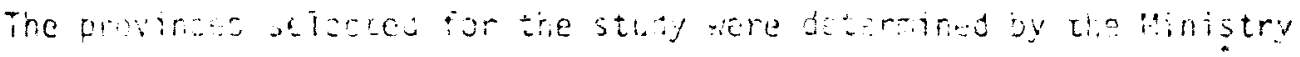

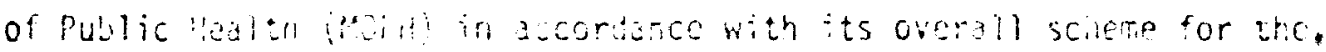
expansion of hed th services into rural areas. Wilnin sach province, existing hasic healin cencers were selected as focal coints for viliagre selection. Waith centers were selecied on the tas is of their length of escablisnment and potential to have had an imact ujon the community as weil as upon the desire to achieve a representative variation of gecgraphical, social and economic conditions within the province. Village selection was accomplished by preparing grid maps of areas adjacent to the BHC and randomly selecting yillages at $1 \mathrm{~km} ., 10 \mathrm{~km}$., and $15 \mathrm{~km}$. from the health center. (Gee map next pace.) Within the village, household selection was done systematicaily from pre-lists of households, using a set pattern of numbering in each village. Within a household, attemsts were made to interview an adult ienale and an acult male preferably, but not exclusivily, a husband and wife. The discreparcy between the rutber of female intervievs conoucted (486) and the nubber of ridic interviews (?27) propesents priniarily the fart that many men were engeged ir agricul blat activities at great distances frow the viliagos during the interviewirg period.

All intervie:ss were carried nut by trained interviewers using initruments which had been sesigned and pretested prior to the survey. (Sce fippendices $A$ and $B, m a l e$ and feir le questionnaires used.) Interviews with fonale re: rondents ricie carried cut exclusively by fomale interviewers. Male interviewers were used for males. In each house.. hold, only a single eliaible respondent of each sex was intervieived resulting in only one wife of a polygnously-married man being incluced in the sample. Each intervicin "required fhetween $_{40} 0$ and 50 minutes to complete. a length which did not affect cloperation, as iudged by respondents" reactions. No efforts ware nade to coerce interviewee cooperation; as an example, in one arca originaliy selected for the sanole, suspicion ano lack of cocpcraticin on the rart. of housetolders made it necascary to seek an aitemative sampla site in order to complete tre rumber of interviews necessary for a ramesentativa sample.

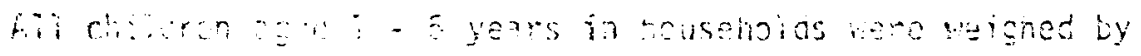




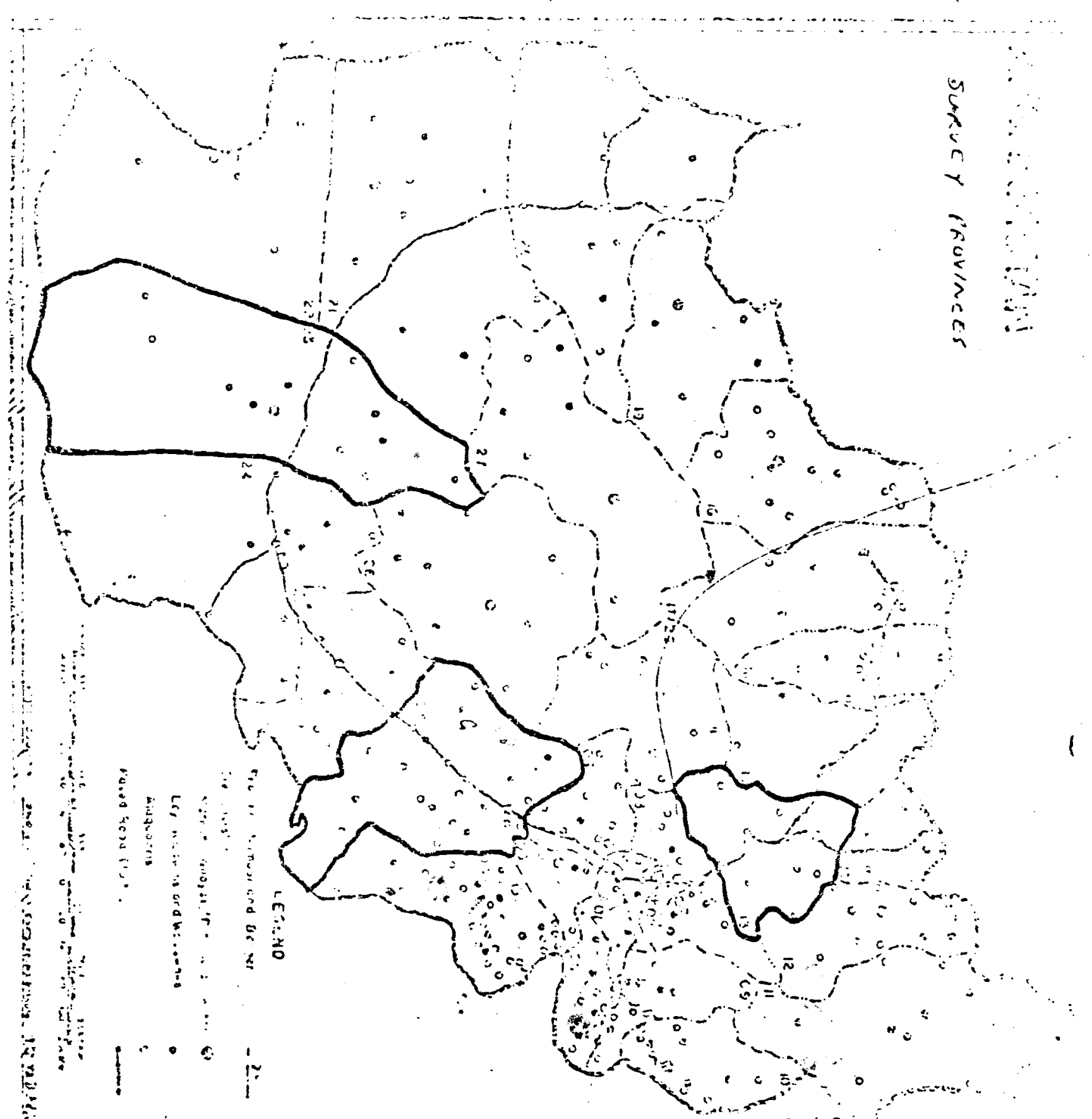

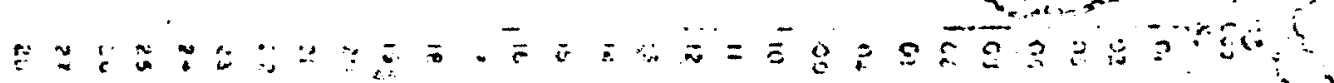

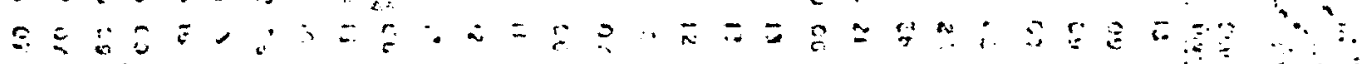

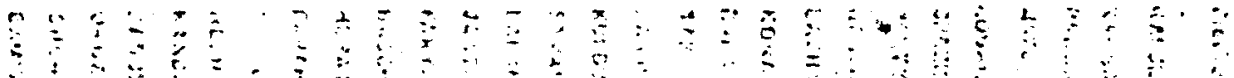




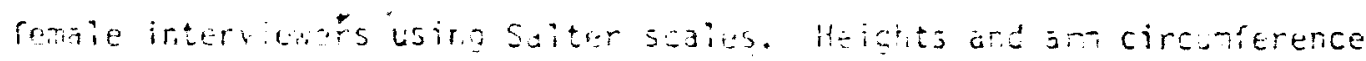
measuremants \% ie carried out cr the same chidren birag siaudardized instruments.

Training of interviewers was conducted over a two week period and included classroom instruction, field interviewing, and evaluation of performance. The practice interviewina also served as a pretesi for the questionnaire. Interviewer performance was quality controlled in the field by team supervisors and by the training staff. The number of intervievis ronducted by village and province during the three munths of fieldwork was:

\section{Number of Intervizws}

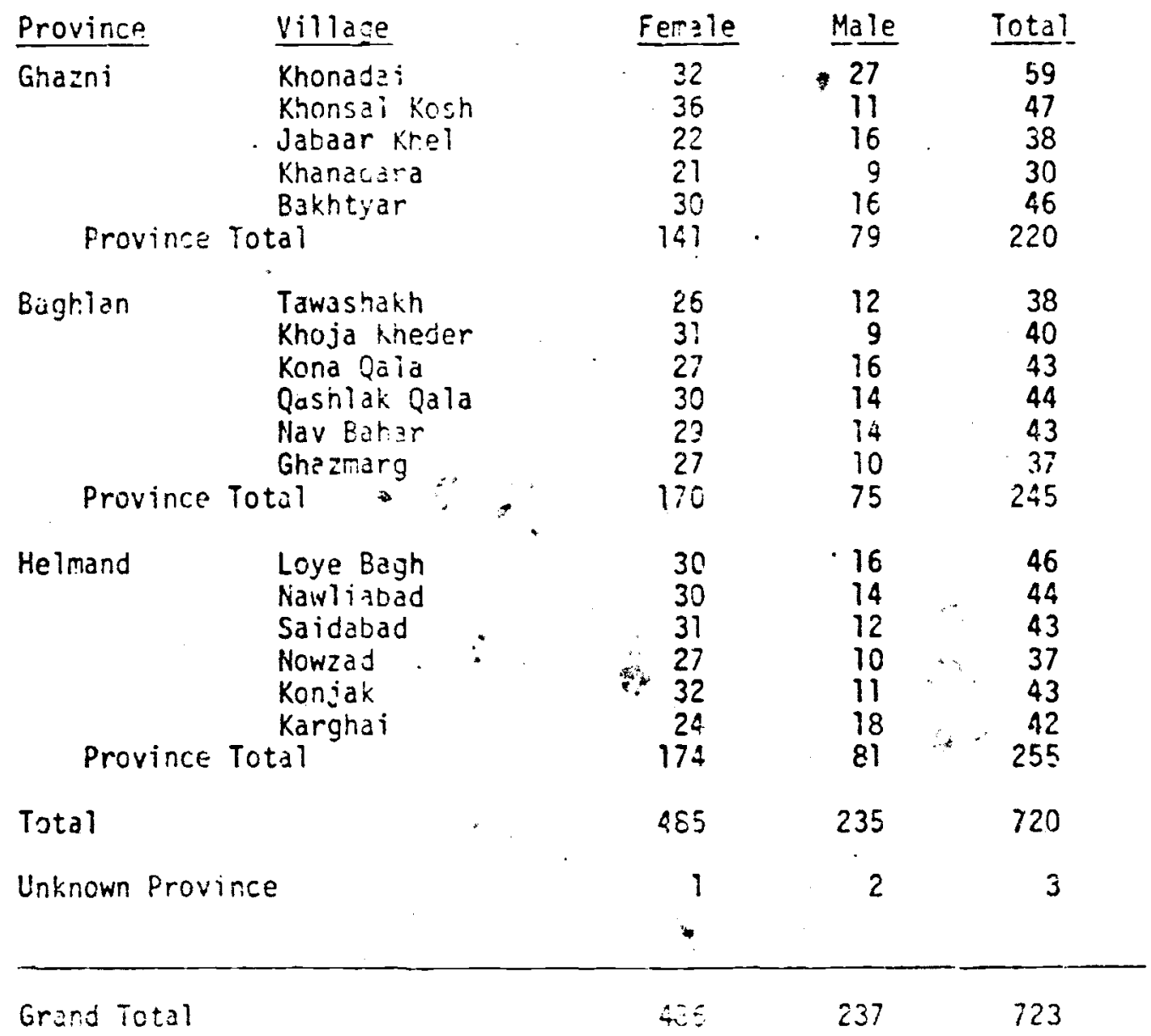


III. Findires

1. The fopulation

A. Characteristics of Resporidents

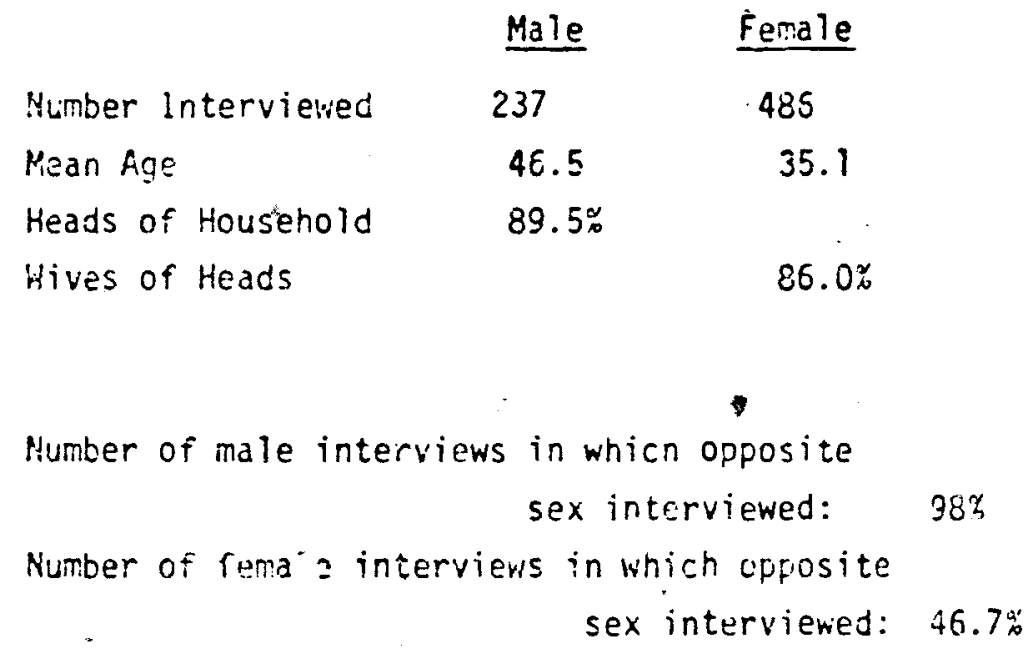

Additionai Wives of Rezpondenis' Husbands

$\therefore \quad \therefore=486$

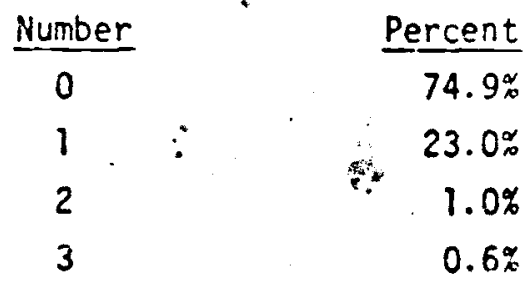

Mean Number Hives/Husband $=1.24$ 


\section{e. Conartiristics ne Mountoles}

i. Distancs of itousehold to Basic Haith Center

$\begin{array}{cc}\text { kns. } & \text { Percent } \\ & 40 \% \\ 10 \mathrm{~km} & 35 \% \\ 15 \mathrm{~km} & 25 \%\end{array}$

2. Individuals in Houschold

\begin{tabular}{|c|c|c|c|}
\hline & \multirow{2}{*}{\multicolumn{3}{|c|}{ Males $\frac{\text { Reported by }}{\text { fertles }}$}} \\
\hline & & & \\
\hline Mean nurber rersons & 7.57 & * & 7.04 \\
\hline Mean number males & 4.08 & & 3.68 \\
\hline Mcan number feniales & 3.25 & & 3.56 \\
\hline
\end{tabular}

3. Individuals Listed in Househola by Ferales

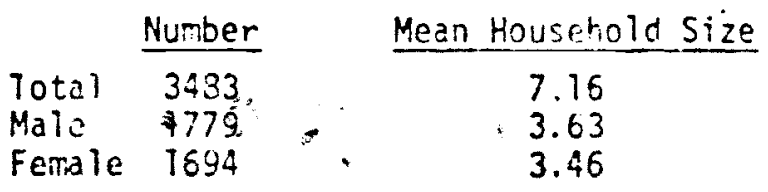

Mean Age of Total Household 21.0

Median Age of :Total Household 14.75

Percent Under 15 jears of age $49.4 \%$

Percent 65 years and over 3.9\%

Deperdency Ratio $114 \%$

Under 15 and over 65

$15-65$ 
4. Age-sex Pyasid

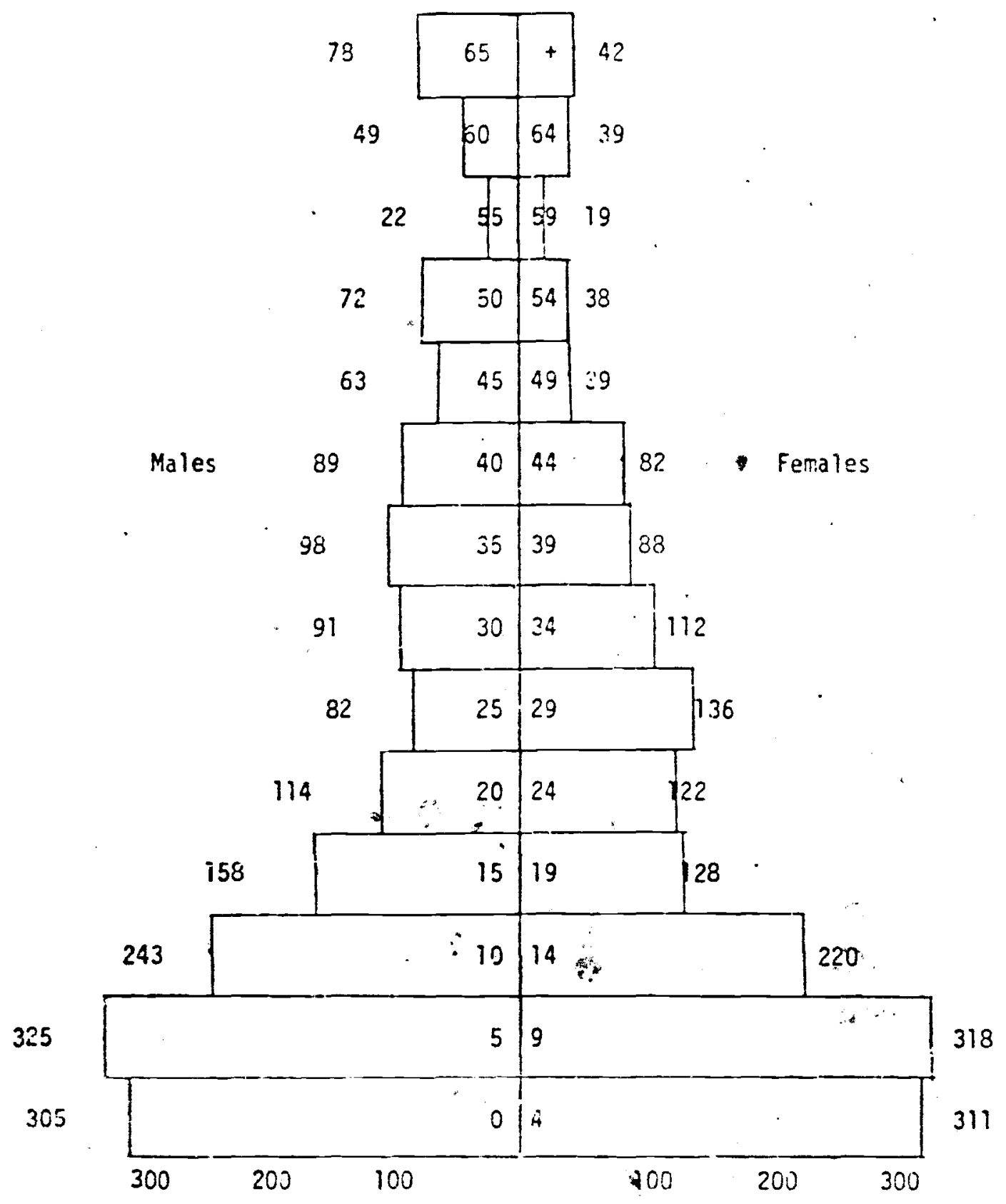




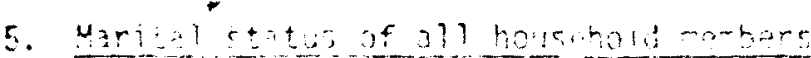

$\begin{array}{lr}\text { stat } & \text { Percert } \\ \text { Fon herried } & 38.3 \\ \text { Hidonde } & 2.5 \\ \text { Nur-arried } & 59.2\end{array}$

6. Mean Years of schoo! ing for persons 15 years of aje and older

$\begin{array}{ll}\text { Maie } & \text { Years } \\ \text { Female } & 0.99 \\ \text { Total } & 1.15\end{array}$

7. Literacy of persons 15 years of age and oider

Male

Female

Total
Percent Literate

3.5

17.6

8. Dccupetion of persons 15 years of age and oider

$\begin{array}{lr}\text { Farmer } & 47.2 \% \\ \text { No Occupation/work } & 12.6 \% \\ \text { Student } & 6.3 \% \\ \text { Laborer } & 5.7 \% \\ \text { Shopkeeper } & 4.3 \% \\ \text { Mullah } & 2.8 \% \\ \text { Officio Wórker } & 2.5 \%\end{array}$

9. Number of Rooms in Household

\begin{tabular}{ccc} 
Number of Roomis & Percent \\
\hline 1 & 38.9 & Mean $=2.25$ \\
2 & 31.1 & Median $=1.85$ \\
3 & 71.8 & \\
4 & 1.3 & \\
5 & 3.8 \\
6 & &
\end{tabular}


$-16-$

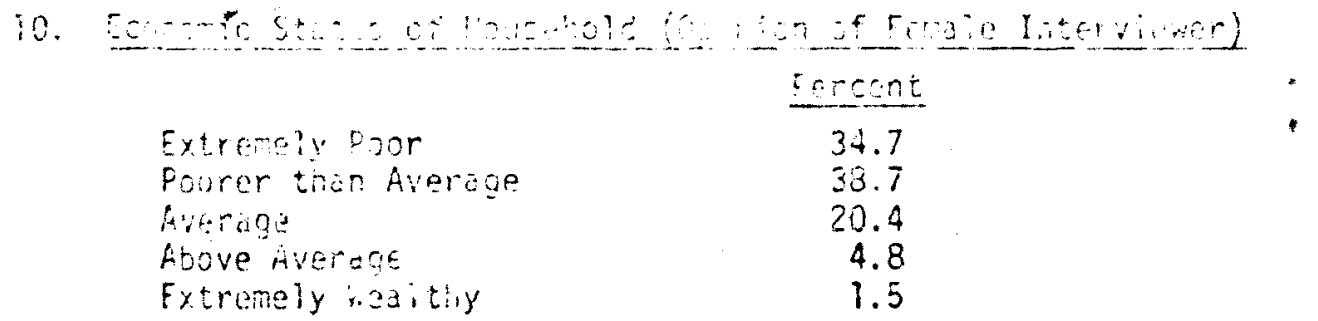


c. Ferribity and "artal:

1. Living Chilcren far Forala Feszordents

Number of Chiliren Percent of Respondents

$\begin{array}{lr}0 & 6.6 \\ 1 & 11.5 \\ 2 & 14.2 \\ 3 & 14.8 \\ 4 & 14.4 \\ 5 & 11.9 \\ 6 & 10.7 \\ 7 & 6.4 \\ 8 & 5.6 \\ 9 & 2.7 \\ 10 & 0.8 \\ 11 & 0.4\end{array}$

Mean Age of Respondents $=35.1$

Mean Numer of Living Children $=3.93$

Median Number of Living Childrer, $=3.37$

Mean liumber of Childr $\cong n$ Ever Born $=5.82$

2. Children Died for Ferale Respondents

$$
N=486
$$

Number of Children Percent of eesconcients

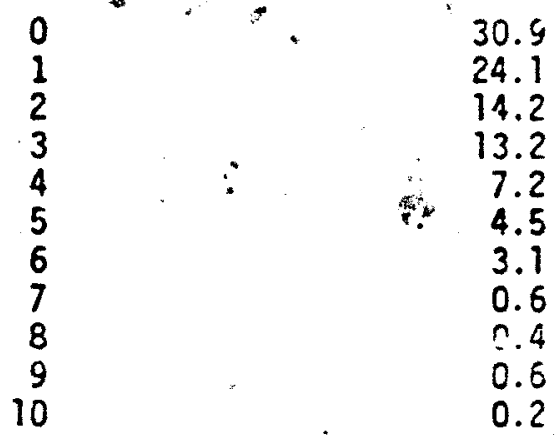

Mean Age of Respondents $=35.1$

Mean ivumber of Chiluren Dejd = 2.83

Median iumber of Chileran Dead $=1.29$ 
3 $\frac{\text { ind }}{23}$

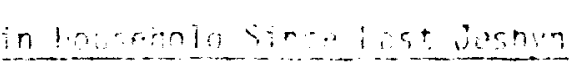

Burver

0
1
2

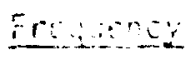

3.8

$1: 7$ prest

67.5

30.3

2.2

Total Births $=169$

Average Births per Howsericid $=0.348$

Births pen 1000 Population $=48.51$

Estiniated Crude Birth fate $=41.5$

4. Marital Age-Specific Fortility Rates

$$
\text { Marital }
$$

Age-Specific Percent Woren

Age Fertility Rates

larried

Age Specific

Fertilitr Rates

$15-19$

$20-24$

$25-29$

$30-34$

$35-39$

$40-44$

$45-49$
440

498

400

373

323

227

27
39.1

79.5

93.4

95.5

91. 5

84.6
172

396

374

356

317

203

23

Total Fertility kate 9226

5. Deaths in Household Sirice Last Jeshyn

Number Frequency Furcent

$\begin{array}{rrr}0 & 401 & 82.5 \\ 1 & 73 & 15.7 \\ 2 . & 10 & 2.1\end{array}$

Total Deaths $=98$

Average Deaths per Household $=0.2$

Deaths per $1000=28.1$

Estimated Crivde Death Re ce $=24.2$

Percent of Deaths Under 5 Years $=59.2$

Infant Mortality Rate $=157$

6.:- Percent of All Dezths linder Five by One-Year Age Intervals

Age

$0-1$

$1-2$

$2-3$

$3-4$

4-5
Percent of Danaths

43.1

29.2

15.5

$3 .:$

8.0 


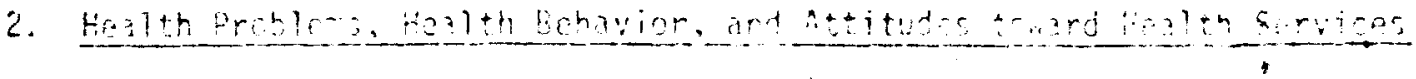

a. Housshold Nembers keported Sick in Last Tho Weeks $=21.6 \%$

Illness Reported:

Respiratory lllness

Colds

Coughs

Black Cough

Pneumoria

TB

Sore Throat

Other

Gastrointestinal Illness

Vomiting

Diarriea

Dysentery

Cholera

Fever

Fever Unspecific

Malaria

Other

Aches and Pains

Stomach Ache

Headachc

Joint Pains

Arthritis :

Other
Percent

2.7

2.1

0.5

3.7

3.2

0.8

5.0

0.8

6.9

4.5

.4

8.8

5.0

0.8
12.6

15.8

14.5

13.9

4.5

3.7

1.5

11.3

Eye Problenis

1.7

Women's Disease :

TOTAL

s.

3.8

83.4

(Note: Mainutrition was named iliness in only $0.7 \%$ of cases.) 
b. Ferent Reportin Bires tho Are still sick t inceviow = 21 e:

c). Primary Treatment Sought for I11-ess

Home Treatrent

Basic Hoalth Canter

Private Doctor Local

Hospital fagional

Pharmacist

Bazaar

Private Doctor Regional

Private Doctoi Kabul

Dokhan

Mullah

Hakim

Cupper

Hospital Kabul $\frac{\text { Percent }}{37.7}$

16.7

8.9

5.5

6.3

5.8

5.2

4.0

3.2

2.8

0.4

0.2

0.4

c2. Satisfied with Treatmont received

Pro - 54\%

Yes - $45 \%$

Satisfaction by Source:

Source

Home

Basic Heal th-Center

Private Doctór Lócal

Hospitai Regional

Pharmacist

Bazaar

Private Coctor:Regiond

Private Doctor Kabul

Dokhan

Percent

49.6

64.7

75.0

71.1

52.4

57.9

53.5

57.1

31.6

Mullat

52.4 


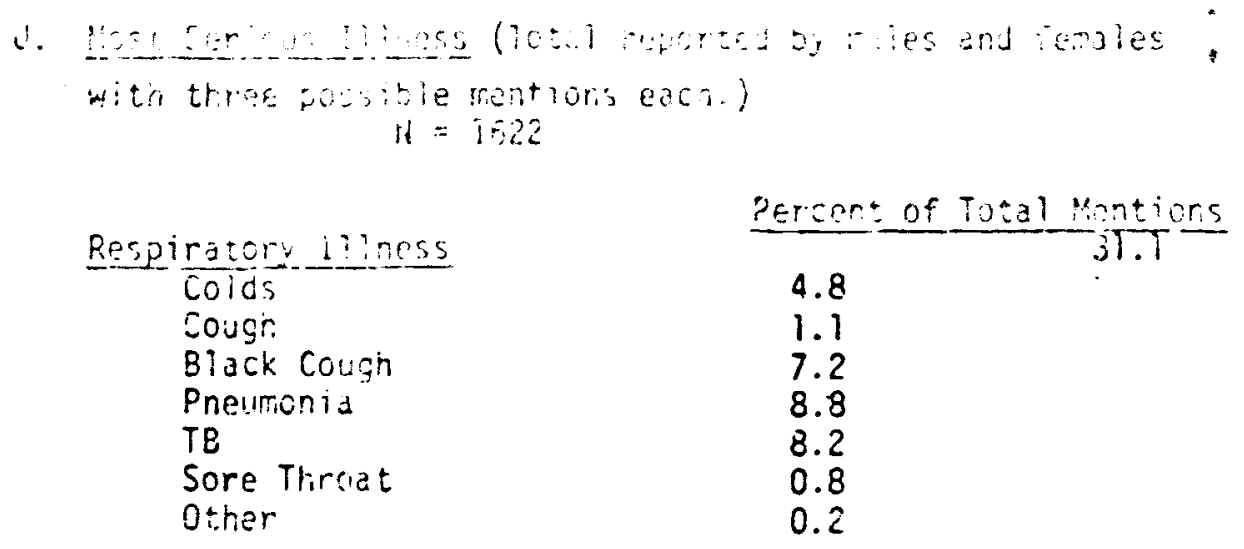

Gascrointestinel Illiness

Vomiting
Diarrioa
Oysentery
Choicra

Fevers

Fever Unspocific.

0.7
8.6
11.8
2.4
7

25.4

Malaria

8.3

other

9.0

3.3

20.6

$\frac{\text { Aches and pains }}{\text { jtomacn uine }}$

Headache

other

3.6

2.3

8.2

Measies

7.5

Eye Protlem $\because:$

2.0

TOTAL

94.8

(Note: Malnutrition mentioned only $0.2 \%$ as most serious illness) 


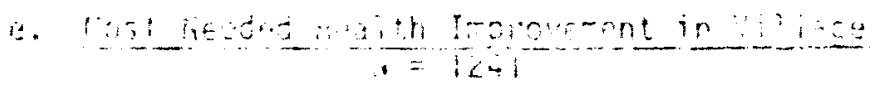

irgoveront singed

Perceni of intal Mentions

? Dostor

24.4

3 Hospital

4 Cicanlinese ir General

$: 0.5$

5 Berter Food

8.9

7.5

6 Pharmacy

3.7

7 Rnads

2.6

TOTAL

84.4

f. Illnesses Associated with A'l Deaths in the Last Year

Illness

Oyseniery/diarrhea

Jinvs

Tuterculosis

Fever.

Swetling

Stonach ache

Black couch

Preumonia/oronchitis

Measles

Heart disease

Unknown

other
Percent of Ali Deaths

15.3

14.3

11.2

8.2

6.1

3.1

3.1

3.1

3.1

3.1

4.1

25.1

99.8

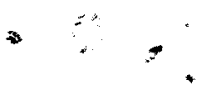

9. Illnesses Associated with Child Deaths by Aze in the Last Year $N=58$

Numbers of Deaths

Illness

Niarrhoaidysentary

Pulmonary/respiratory

Fever

Measles

Other

Unknown

TOT:L

\begin{tabular}{|c|c|c|c|c|c|}
\hline & & Ane & & & \\
\hline$\overline{0-1}$ & $7-2$ & $2-3$ & $3-4$ & $A-5$ & Tot \\
\hline 9 & $\begin{array}{l}0 \\
5\end{array}$ & 3 & 0 & 0 & \\
\hline 2 & 3 & 2 & 0 & $i$ & \\
\hline 2 & 0 & 2 & 0 & 0 & \\
\hline 0 & 1 & 1 & $i$ & 0 & \\
\hline 5 & 7 & 1 & 0 & $?$ & \\
\hline 3 & $i$ & 0 & 0 & $\underline{0}$ & \\
\hline 25 & 17 & 9 & 2 & 5 & \\
\hline
\end{tabular}




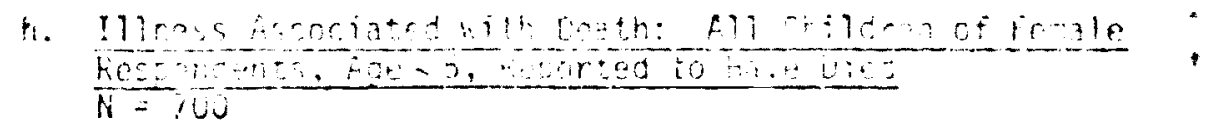

\begin{tabular}{|c|c|c|c|c|c|c|c|}
\hline & \multicolumn{5}{|c|}{ Numbers of Ce?ths } & \multirow[b]{2}{*}{ Total } & \multirow[b]{2}{*}{ Percent } \\
\hline $111 n$ & $\overline{0-1}$ & $T=\overline{2}$ & $2-3$ & $3-4$ & $\overline{4-5}$ & & \\
\hline Jinns & 136 & 35 & 22 & 6 & 8 & $20 \%$ & 29.6 \\
\hline Dizrrhea/Dysentery & 19 & 17 & 29 & 7 & 6 & 78 & $\$ 1.2$ \\
\hline Black Cough & 21 & 12 & 15 & 11 & 2 & 61 & 8.7 \\
\hline Measies & 11 & 6 & 21 & $\cdot 12$ & 9 & 59 & 8.4 \\
\hline Pneumonia/Bronchitis & 33 & 13 & 6 & 4 & 2 & 58 & 8.3 \\
\hline Fever & 19 & 6 & 10 & 1 & 2 & 38 & 5. \\
\hline Smalloox & 5 & 6 & 10 & 7 & 4 & 3? & $\$ .6$ \\
\hline Injury & 6 & 0 & 3 & 1 & 2 & 12 & 1.6 \\
\hline Malnutrition. & 15 & 0 & 3 & 0 & 0 & 18 & 2.6 \\
\hline other & 86 & 14 & 15 & 14 & 8 & 137 & 19.6 \\
\hline Total & 351 & 109 & 134 & 63 & 43 & 700 & \\
\hline Percent & 50.7 & 15.6 & 19.1 & .9 .0 & 6.1 & & \\
\hline
\end{tabular}

i. Number of Dais (nidwives) in Female Respondent's Village

\begin{tabular}{cc} 
Number & Respondents \\
\cline { 2 - 2 } 0 & 57.4 \\
1 & 13.8 \\
2 & 10.1 \\
3 & 2.1 \\
4 & 0.8 \\
Unknown & 15.8
\end{tabular}

j. Use of Dai by Female Householders

$$
\begin{aligned}
& \text { No - 51.5\% } \\
& \text { Yes - 48.5\% }
\end{aligned}
$$

k. Residence of Dai

$$
N=189 \text { (temale }
$$

$$
\text { respondents) }
$$

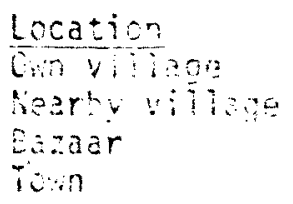

$$
\begin{gathered}
\text { Fercent } \\
\hline 09.9 \\
8.5 \\
0.5 \\
1.1
\end{gathered}
$$


$-19$.

1.

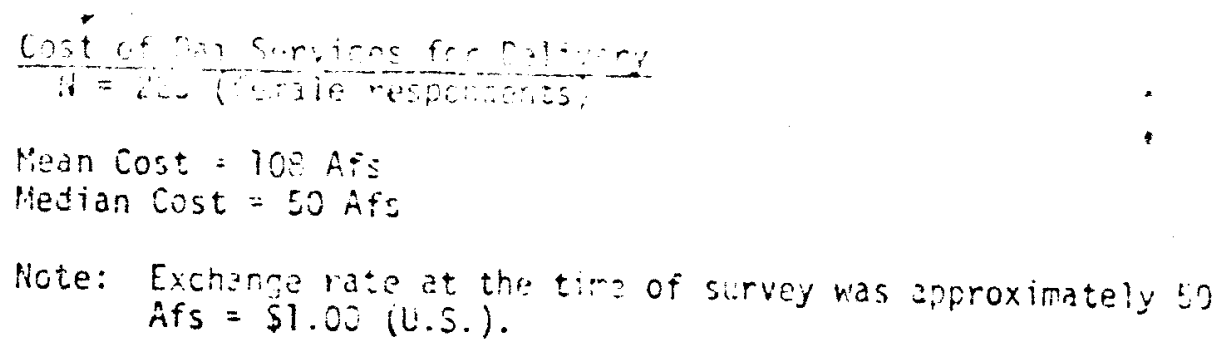

m. For Women Riot Reporting lyse at Da:

If dali does not assist in delivery, who does?

$N=225$

Women in ilousenold

Women Outside household

All at Health Center

Regional Hospital

Kabul Hospital $\frac{\text { Percent }}{93.9}$

1.7

0.3

1.0

0.3

2.7

n. Satisfaction with Assistance Received at Delivery $P:=472$ Female Respondents

$\begin{array}{lr} & \text { Percent } \\ \text { Very Satisfied } & 5.6 \\ \text { Satisfied } & 50.8 \\ \text { Intermediate } & 30.9 \\ \text { Unsatisfied } & 8.9 \\ \text { Very Unsatisfied } & 2.8\end{array}$

o. Percent of Household Who Have Visited BHC

$$
\begin{aligned}
& N=2.31 \text { Males. } \\
& \quad 476 \text { Females } \\
& \text { Male } \quad 51 \% \\
& \text {. Fen ale } 67 \%
\end{aligned}
$$

?. 


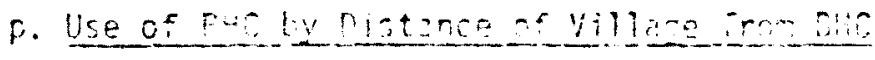

$N=476$ Fenale Respondents

\begin{tabular}{cc} 
Distance frcm villaze & Percent Used Ble \\
\hline $1 \mathrm{~km}$. & $75 \%$ \\
$10 \mathrm{~km}$. & $61 \%$ \\
$15 \mathrm{~km}$. & $63 \%$
\end{tabular}

q. If Used 6 ب.C

What do You Think of the Q11ality of Modicine and Services of the BHC?

Male $(N=340)$ Female $(N=289)$

$\begin{array}{lrr}\text { Very Good } & 7.9 \% & 9.3 \% \\ \text { Gcod } & 50.0 \% & 48.8 \% \\ \text { Intermediate } & 17.9 \% & 17.3 \% \\ \text { Poor } & 23.6 \% & 24.0 \% \\ \text { Very foor } & 0.7 \% & 0.3 \%\end{array}$

r. What Do You Think of trie Personal Treatmeni You Receive at the BHC?

\begin{tabular}{lcc} 
& Male $(N=144)$ & Female $(N=286)$ \\
\cline { 2 - 3 } Very Good & $11.8 \%$ & $11.5 \%$ \\
Good & $59.0 \%$ & $52.8 \%$ \\
Intermediate & $14.0 \%$ & $18.2 \%$ \\
Poor & $9.7 \%$ & $17.5 \%$ \\
Very Poor & $4.9 \%$ & 0
\end{tabular}


s. Prirary Reasen biv fue no used

Po one sick

Services in torr. better.

lio medicines avaibule

Hio money

Family cbjection

Too far away

Go to private docter

Staff requires money

Too expensive

Heard bad things

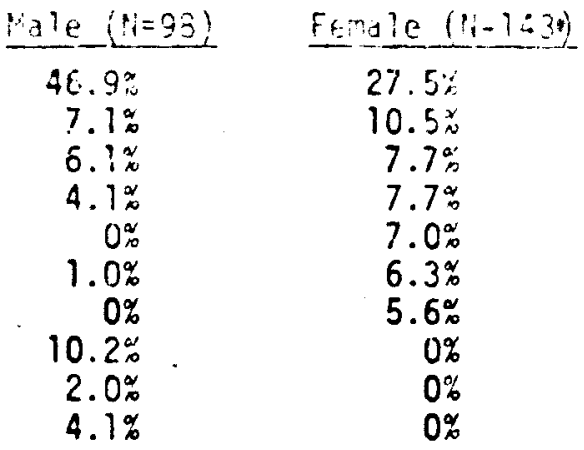

t. Where do you receive the best treatment for an illness which canrot te treuted at honie?

Village $\frac{\text { Male }(i v=227)}{2.6 \%} \quad$ Female $\frac{(i=458)}{0 \%}$

Pharmacies

$0.9 \%$

$5.3 \%$

$3.4 \%$

Mullins or Shrines

$34.8 \%$

$3.1 \%$

$25.0 \%$

$\mathrm{BHC}$

Loca? Private Doctor

Regional Private Doctor

$8.8 \%$

$7.0 \%$

$7.0 \%$

$29.1 \%$

$0.1 \%$

$33.3 \%$

$8.8 \%$

$14.5 \%$

2. $6^{\circ}$

$6.4 \%$

3. $0 \%$

$3.0 \%$

u. What is your best estimate of the total amount of money which your householdascerit on health sorvices since Jeshyn last year?

Mean Year Expenditures Median Year Expencitores
Male. 2878 Afs 1000 Afs
Female

3873 Afs

1999 Afs 
v.

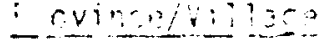

Gnazni

khorada?

Khorisal Kest:

Jabaar Khiel

Khariadar

Bakhtjar

Baghlan

Tawashakh

Khoja Khecier

Kona $\mathrm{S}_{\mathrm{a}} \mathrm{l}$

Qushizk Quia

Nav Bahar

Ghazriara

Helmand

Loye Bejh

Nawl iabaj

Saidabad

Nowlac

Konjak

Karghai

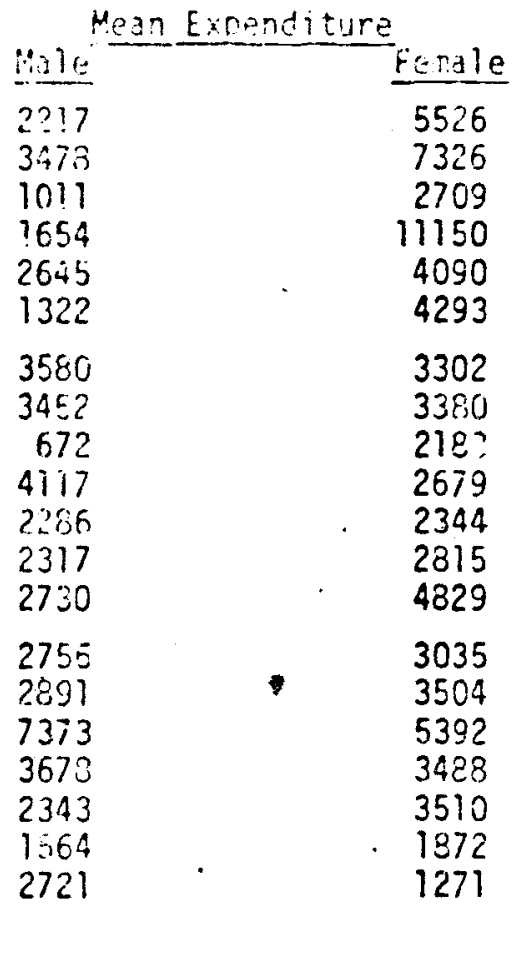

w. What is your coinion of the drugs which you can get in tha dokhan (viliage shon)?

$\begin{array}{ll}\text { Good } & \text { Percent } \\ \text { Average } & 26.8 \\ \text { Poor } & 27.4 \\ & 45.2\end{array}$

$x$. Do you get advice from arvone when a finily member is sick? $N=235$ (male)

No. $-84.4 \%$

y. Of those who sot advice. What is the sex of the person you set adure trin:

Eersent

$\begin{array}{lr}\because 12 & 4.7 \\ \text { remale } & 8.3\end{array}$




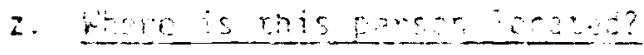

Own viliage

iteorby village

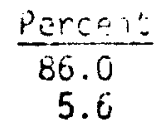

a. Qces tr.e person who gives advice also give medicine? No $-76.3 \%$

b. Do you krow sonerne in your illage who can tive you an injection? Maic Resomendents Farale Resporients $(v=205)$ $(N=481)$

$$
\text { Yes }
$$

$$
37.1 *
$$$$
58.6 \%
$$

Sex of Injector is Male

$96.0 \%$

$32.0 \%$

Injector coes no: give recicine

$86.7^{\circ}$

c. Do you think children are healthier or less healthy than 5 vears roo? Male Respondents (il=22Q) Fenale kespondents $(i=436)$

Healthier

Same

Less Healthy
63.6

15.6

20.9
38.1

23.9

39.1

d. Do you think that more or fewer chiloren die now than j years ano? Male Resoundents ( $N=228$ ) Eemale Rospondents ( $N=436)$

More

Samie

Fewer
11.6

21.3

57.1
28.3

14.8

50.9

\begin{tabular}{|c|c|c|}
\hline & Hie is (220) & $60-3] \leq 80$ \\
\hline fetter & 0.2 & 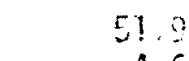 \\
\hline $52 m a$ & 12.0 & \\
\hline lorse & 0.4 & is \\
\hline $50 n^{\prime} t$ now & 9 & \\
\hline
\end{tabular}

e. Do vou thing thit health services are better ur worse then byears aro? 
3. Child fozring Practices

How long do you br - ist feed your boy tabies? (it = 474 reales)

Mean Length - 23.5 Months

How long do you breast feed your girl babies? ( $N=474$ Fonales)

Mean Length - 23.9 Months

At what age do you begin to feed your child foods in addition to milk? $\quad(N=475$ Fenales $)$

Mean Age 11.5 Months

What are the best foods to tegin feeding your babies?

First Mentioned Food (452) Second Mentioned Food (434)

Bread 17. $\%$ Milk 10.4\%

Milk 16.6\% Rice

Rice 10.5\% Bread 7.0\%

Soft Rice $8.6 \%$ Yogurt $6.5 \%$

Bread and Tea 6.0\% Soft Rice. $6.5 \%$

Vegetable Soup , 4.4\% Vegetable Soup 4.9\%

$\begin{array}{lll}\text { Yogurt } 2.2 \% & \text { Butter }\end{array}$

At what age do you first yive your baby these foods:

Soft Meat

Mean Ace Manths, ( $N=486$, Females)

Eggs

Bread

Tea

Fruits

Vegetable
20.2

26.3

11.3

11.7

16.5

18.8 
Hon often do you aive your crileran powated milk?

Hever

fereat $\quad(i)=475)$

Alout Once/Moith

79.8

Abou: Onceltrek

0.8

Three to Four Times Week

6.5

Every Day

2.3

10.5

How often do you sive your children animal milk?

Never

About Once/Month

Abcut Cnce/Week

Three to Four Times Week

- Every Day

$$
\frac{\text { Percent }}{28.8}
$$

2.3

20.5

7.0

41.4
$(N=413)$

nuring your last pregnancy did you take ary medicines to prepare you for a healtny baby? (it = 475).

$\begin{array}{ll}\text { No } & 97.1 \% \\ \text { les } & 2.9 \%\end{array}$

Sources of Mecicine:

Woman in Village

Pharmacist

BHC

Private MD Local

Private MD Regional

$$
\frac{\text { Percent }}{15.4} \cdot \quad(i 1=13)
$$

23.1

7.7.

$23.1^{*}$

15.4

After your last baby was born dif you get advice from anyone outside your household on hew to keep it healthy? (N=480 Females)

$$
\begin{aligned}
& \text { No }-\$ 6.9 \% \\
& \text { Yes }-3.1 \%
\end{aligned}
$$


Source isotsond:

\begin{tabular}{|c|c|}
\hline & Wunger lonticrod \\
\hline Hakinj: & 6 \\
\hline Dai & 3 \\
\hline Other woman & 3 \\
\hline Others & 3 \\
\hline
\end{tabular}

In your opinion what is the rost important thing to do to keep your baby healthy?

Mdie Respondents (155) Female Respondents (397

Clean Food and Water

Consult Good Doctor

Good Food

Vitamins

Heal tin Personnel Near Village

cleanliness
$14.8 \%$

$11.6=$

$11.0 \%$

9.7\%

$9.7 \%$

$5.8 \%$
$35.3 \%$

0

$12.3 \%$

$53 \%$

0

$49.7 \%$

Mave you ever treated any of your children for diarinea?

$\begin{array}{lcc} & \text { Males }(233) & \text { Females }(467) \\ \text { No } & 41.2 \% & 14.4 \% \\ \text { Yes } & 52.8 \% & 85.6 \%\end{array}$

Should, ou feed yourocille when he has diarrhea?

$\begin{array}{lrcc} & \frac{\text { Males }(233)}{30} & & \text { Females }(457) \\ \text { No } & 33.0 \% & & 27.4 \% \\ \text { Yes } & 66.5 \% & & 70.0 \% \\ \text { Don't Know } & 0.4 \% & & 3.6 \%\end{array}$

Should you give your child water when he has diarmea?

Males (234)

Ho

2). $8 \%$

Yes.

$78.2 \%$ 
Whot ame rost foods for a citid with diarried?

Sort sice

Soft rice and $0 \% 1$

Nothing

hater

Bread

Chesse, Yogurt, Qurbot

Bread and Tea

Soup Végetable
Malesitic

$32.6=$

$2.3 \%$

$7.0 *$

$5.27 \%$

4.1\%

$4.1 \%$

$1.1 \%$

$0.8 \%$
Ferales (15)

$37.0 \%$

0

$.3 \%$

$1.0^{\alpha}$

$12.7 \%$

$2.2 \%$

$11.7_{\%}^{\circ}$

$3.5 \%$

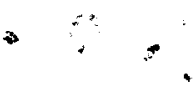


$-?]-$

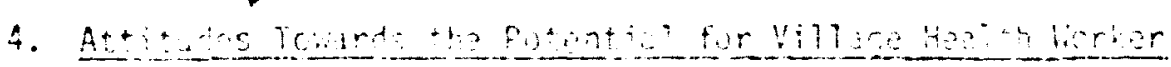

Respondent's pinion on feasibility of village health worker in hisitior village.

$\begin{array}{lcc} & \text { Males }(235) & \text { Females }(483) \\ \text { Not Feasible } & 17.4 \% & 3.7 \% \\ \text { Feasible } & 78.3 \% & 95.2 \% \\ \text { Don't know } & 4.3 \% & 10 \%\end{array}$

If not feasible, why not?

Person not available

No one learned enough among us

People are poor

Not feasible for this village

Medicine and doctor good enough

Men wont permit it
Males (36)

$33.3 \%$

$25.0 \%$

$8.3 \%$

8. 3 :

2. 8\%

0
Females (6)

$28.6 \%$

0

$14.3 \%$

$14.3 \%$

$? .3 \%$

$14.3 \%$

F. 


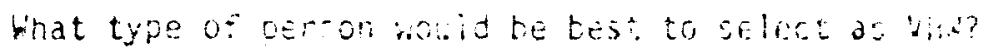

4ze $\begin{array}{ll}\text { Young } \\ \text { Middie-figed } \\ \text { older } \\ \text { Don't know }\end{array}$

$\underline{\text { Sex }}$

Male

Female

Both
Males 1193

Education Viean Vears Education

Literacy Reguired? Yes

Should person have experience outside village?

Eest Qualities for VHW

Good character and patience

Proper marner

Muslim and humanitarian

Experienced

Clever and irtelligent

Good person

How should VHid be selected?

By the people

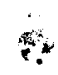

By the goverrment

By governient ano peopie

By village leade:

Ev government and village leader

should be choser fron arong injectionists

Ey leaders and sooplo
Yes $04.3 \%$
23.5\%

$21.1 \%$

$5.1 \%$

$30.8 \%$

Males!los) Ferales (42).

$\begin{array}{rl}13.7 \% & 11.6 \% \\ 6.3 \% & 43.2 \% \\ 80.0 \% & 44.9 \%\end{array}$

Males (198) Fema?es (421)

$98.4 \%$

11.7 years

$100 \%$

$81.3 \%$

$10.7 \%$

$37.7 \%$

$38.9 \%$

$8.0 \%$

$43.2 \%$

$44.9 \%$

9.8 years$$
1.35
$$

$\begin{array}{cc}\text { Males }(183) & \text { Fem } \frac{3 ! e j i(2)}{30.5 \%} \\ 40.4 \% & 2.6 \% \\ 15.3 \% & 4.5 \% \\ 10.4 \% & 1.0 \% \\ 8.2 \% & 28.1 \% \\ 6.6 \% & 2 i .6 \% \\ 1.0 \% & \end{array}$




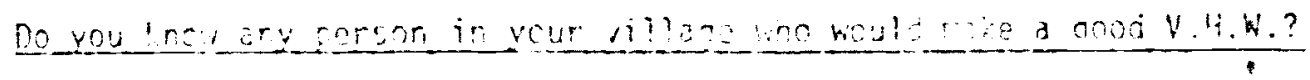

Males 1220$)$ Females $(460)$

$\begin{array}{lll}\text { No } & 73.2 \% & 82.8 \% \\ \text { Yes } & 26.8 \% & 17.2 \%\end{array}$

Of person rentioned

Mean Age

$33 .:$ years

31.5 years

Sex

Male

$91.4 \%$

$61.0 \%$

Female

$8.6 \%$

$37.7 \%$

Education - Mean Years

$9.2 \%$

$7.9 \%$

Literacy

$82.8 \%$

$72.0 \%$

Males

Females

Experience outside villace required.

$63.8 \%$

$61.5 \%$

Why would this person mate a good Vtit?

Males (52) Females (71)

Relative of villaçe chies.

0

$4.2 \%$

Relative of respondent

0

$8.5 \%$

Has no other work

0

$1.4 \%$

People like him/her

$38.0 \%$

$15.5 \%$

Person is useful ":

12.0\%

$2.8 \%$

knows how to give injections

$4.0 \%$

$1.4 \%$

Good education

$16.0 \%$

$16.9 \%$

Person will heip

0

$5.6 \%$

Person is informed

?

$14.0 \%$

$4.2 \%$

Person is clever

$14.0 \%$

If there was a person in the village who provided medicines and whose job it was to inprove health, should he/she bo gaid?

- Males (217)

Wo

$9.2^{*}$

Yes

80.2:

Dan'i kros

10.6 
Ho. stould in te raid:

Governivent should provide salary

Pecple sriculd provide salary

Pecple could not provide salary

People should provide part of salary
Males (136)

$27.1 \%$

$43.4 \%$

$19.9 \%$

$6.6 \%$

Could a woman. frum this village be trained to work on village heal th problems if trairing vere nearby ard she could return home in evenings from tra in ing?

$\frac{\text { Males (220) }}{29.5 \%} \quad \frac{\text { Fenales (376) }}{10.1 \%}$

Same Question with training farther akirk?.

$$
34.1 \% \quad 7.4 \%
$$

Male Respondents, would vou allow vour wife or daunter to be trained to work on village heal th problens?

$$
\text { Yes } 26.8 \% \quad(H=142)
$$


5. Compricatone in visen

For fendes, Do you over listen to the radio? (455)

$$
\begin{aligned}
& 10-52.7 \% \\
& \text { Yes - 47.j }
\end{aligned}
$$

Of these woren who isten, how many times a week do you listen? ( $N=245)$

\section{Percent}

\section{Less than 1}

13.5

1

4.1

2

11.0

3.

5.7

4

1.2

5

0.4

6

1.6

7

62.4

\begin{tabular}{|c|c|c|}
\hline & & Percent \\
\hline & Our Honie & 66.7 \\
\hline & Friends Home & 24.3 \\
\hline & Relatives Home & 3.6 \\
\hline & other place in village & 5.0 \\
\hline & Bazaar & 0.5 \\
\hline Which program & s most interesting? (N= & \\
\hline & Stories & 2.8 \\
\hline & Farmers' Program & 3.7 \\
\hline & Radio & 2.3 \\
\hline & News & 9.7 \\
\hline & Music & 62.0 \\
\hline & Fanily Life & 19.4 \\
\hline
\end{tabular}

Where is radio located that you listen to? 


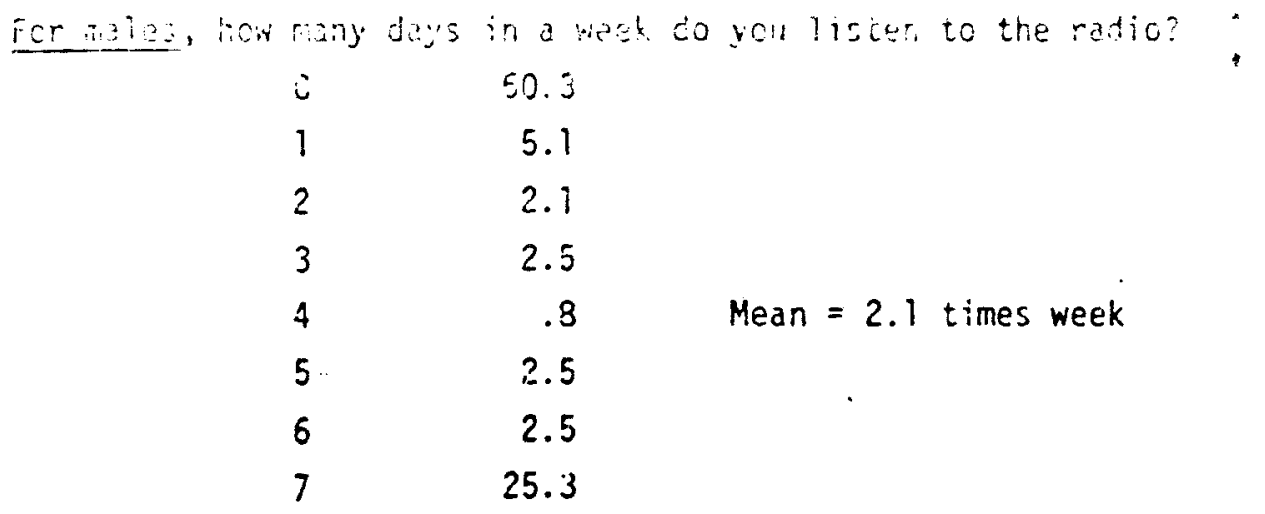

$\begin{array}{cc}\text { Men's Favorite Programs } & (N=109) \\ \text { Stories } & 1.8 \\ \text { Farme: 's Progran } & 27.5 \\ \text { Ráio } & 6.4 \\ \text { News } & 33.0 \\ \text { Husic } & 19.3 \\ \text { Family Life } & 5.5\end{array}$

Location of Radio Men Listen To

$\begin{array}{lr}\text { Our home } & 64.9 \\ \text { Friends home } & 14.4 \\ \text { felatives home } & .9 \\ \text { Other place in village } & .9 \\ \text { Bäzaar } & 18.0\end{array}$

Asked of Males

Would it be permissable for your wife to go to the following places?

\& Would Allow"

$\begin{array}{lr}\text { Female friends in village } & 41.8 \\ \text { Bazaar } & 7.8 \\ \text { Basic iealth Ceniter } & 12.9 \\ \text { Shrine } & 6.5\end{array}$




\section{Aereros romios}

Do you have your husbend's permission to 30 to ary of the foilowing places without a conpanion?

$\begin{array}{lr}\text { Ferale Friends in Village } & \text { o With ior issicn } \\ \text { Bazaar } & 3.3 \\ \text { Basic Health Center } & 6.7 \\ \text { Shrire in Village } & 44.3 \\ & 19.0 .\end{array}$

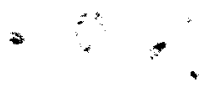


6. Sention (Male Rusurwencs driy)

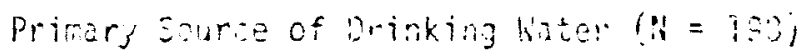

Well in fara 22.9

well in village 1.5

River $\quad 5.9$

Jui $\quad 37.8$

Spring $\quad 12.8$

Kariz 19.1

Satisfied with water supply?

Yes - 78. $1^{\circ}$

Would you contrihute your labor to improve the drinkirg supply of your village if government specialists were avilable?

Yes - 86. $1 \%$

Would you contribute financially to inprove the water suppiy in your village, if government specialists were avallable?

Yes $-60.3 \stackrel{.}{\sim}$

Primary Sanitation Used by Household.

1. Latrine in house 3.7

2. Latrine in yard, 39.2 .

3. Deep hole in yiard 3.7

4. Deep hole outside yard 15.3

5. Hole in yaro $: 0.5$

6. No facilities 27.0 ?

Are you satisfied with the saritation facilities availns?

$$
\text { Yes - 54.5\% }
$$

Wiiling to help with labor to improve?

Yes - $81.4 \%$

Whling to help with money to irprove?

YES - $48.4 \%$ 
7. Attiturestowert Fomilycizo

How many more children would you like to have?

\begin{tabular}{cc} 
Males $(i 18)$ & Females $(333)$ \\
\hline $63.5 \%$ & $65.4 \%$ \\
$2.7 \%$ & $3.3 \%$ \\
$10.1 \%$ & $7.8 \%$ \\
$5.9 \%$ & $10.8 \%$ \\
$1.6 \%$ & $3.0 \%$ \\
$5.0 \%$ & $3.3 \%$ \\
$1.6 \%$ & $4.5 \%$ \\
$4.2 \%$ & $6.9 \%$ \\
$5.0 \%$ & $0.7 \%$
\end{tabular}

Mean Number

Additional

Children Desired

I.E

1.2

Number of Additicnal Boys lianted

\begin{tabular}{crrr} 
Number & $\frac{\text { Males }}{53.6 \%}$ & $\frac{\text { Females }}{60.4 \%}$ \\
\hline 0 & $\ddots 7.2 \%$ & $5.3 \%$ \\
2 & $13 \%$ & $12.5 \%$ \\
3 & $6.5 \%$ & $13.6 \%$ \\
4 & $5.8 \%$ & $3.1 \%$ \\
5 & $10.9 \%$ & $3.6 \%$ \\
6 & $2.1 \%$ & & $1.2 \%$ \\
& Mean $=1.46$ & Mean $=1.11$
\end{tabular}




\begin{tabular}{ccc} 
Number & Nucber of ititional Girlicianted \\
\hline 0 & $\frac{\text { Males }}{67.3 \%}$ & $\frac{\text { Females }}{79.6}$ \\
1 & $9.0 \%$ & 9.3 \\
2 & $9.6 \%$ & 6.6 \\
3 & $7.1 \%$ & 3.7 \\
4 & $1.3 \%$ & 0.5 \\
5 & $5.1 \%$ & 0.3 \\
6 & $0.6 \%$ & 0.0 \\
& Mean $=0.85$ & Mean $=0.37$
\end{tabular}

Number of Additional Children Wanted by Female Respondents

$$
N=333
$$

Number of Live Children

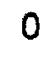

1

2

3

4

5

6

7
Number of Additional Children ilanted

$2.45 \%$

$3.20 \%$

$2.92 \%$

$1.53 \%$

$0.74 \%$

C. $64 \%$

$0.46 \%$

$0.17 \%$
Age of Female $R$

$$
\begin{aligned}
& 15-19 \\
& 20-24 \\
& 25-29 \\
& 30-34 \\
& 35-39 \\
& 40-44 \\
& 45-49
\end{aligned}
$$

Mean Noper of Additional Children lianted

3.91

2.96

2.47

1.53

0.98

0.64

0.50 
Hould you be interested in learning atout vaye chet would allur you to ircrease the amount of time betweer prandricies?

vales (210) Fencies (323)

Would be interested

$69.0 \%$

$91.6 \%$

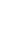




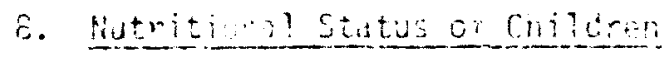

Aim. Circunferenco Measurament: Chiltran $<5$ years

$\begin{array}{rrr}\text { Green } & \text { Yellow } & \text { fied } \\ 31.2 \% & 35.7 \% & 22.1 \%\end{array}$

Al! Children (Age < 5)

Arm Circunference

Sick in last two weeks

Green Yellow Red

Sick recently but recovered

$17.8 \%$

30.2

51.9

(iv $=129$ !

sick recently and still sick

$35.0 \%$

$30.0 \%$

$35.0 \%$

(N $=20$ !

liot sick in last two weeks

$15.9 \%$

$30.8 \%$

$53.3 \%$

$(N=107)$

$34.8 \%$

$40.0 \%$

$25.2 \% \quad(N=330)$

All Cnijuren (Age 5)

Arm Circumference

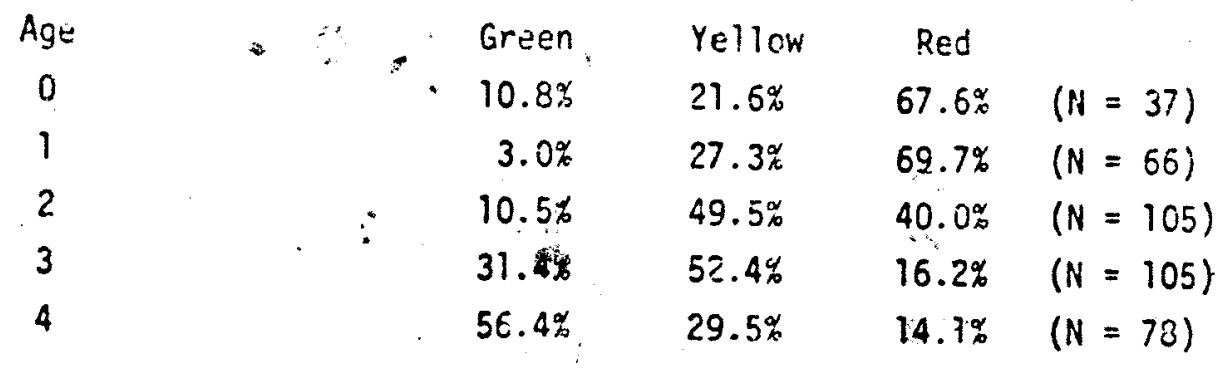




\begin{tabular}{|c|c|c|c|c|}
\hline & Arr Circumiererco & & & $\cdot$ \\
\hline Age & Green & Yei icin & Ped & \\
\hline 0 & $5.9 \%$ & $29.4^{\circ}$ & $64: 7 \%$ & $(N=17)$ \\
\hline 1 & $6.1 \%$ & 33.28 & $00.6 \%$ & $(i)=37)$ \\
\hline 2 & $13.7^{\circ}$ & $58.3 \%$ & $27.5 \%$ & $(N=51)$ \\
\hline 3 & $35.2 \%$ & $51.7 \%$ & $12.1 \%$ & $(N=58)$ \\
\hline 4 & $53.3 \%$ & $33.3 \%$ & $13 .: \therefore \%$ & $(N=30)$ \\
\hline
\end{tabular}

Girls (Age< 5)

Arm Circumference

Age Green

Yellow

Red

0

$15.0 \%$

$15.0 \%$

$70.0 \%$

$(N=2.0)$

1

$0.0 \%$

$21.2 \%$

$78.8 \%$

$(N=3 \hat{3})$

$7.4 \%$

- 4U.7\%

$51.9 \%$

$(1)=54)$

$25.5 \%$

$53.2 \%$

$21.3 \%$

$(N=47$ !

4

$58.3 \%$

$27.1 \%$

$14.5 \%$

$(N=48)$

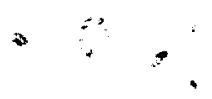


i..

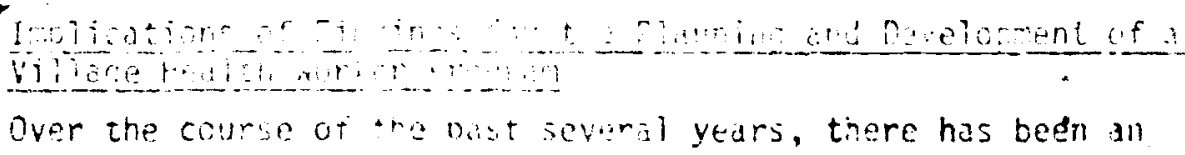

increasing awarensss that a vist majority of health problems encountered in rural settings of develasung countries may be adequately treated or prevented by iraking use o: local resources. The "sarefoct Doctor" of China is the most frequently mentioned example of success with this approach. (For other examples, sce "A Reviow of Alternative Approuches to Health Care Delivery" prepared by MSH:)

Regardiess of the success of the illlage health worker concept in several countries, the planning and development of such a program is not simple, and lack of proper planning and understanding for the envircnment in which a village health worker (VHW) serves has, on several uccasions, led to failure. A list of information helpful, if not essential, to the planning of a VHid program is long and includes such questions as:

- the most prevaient illnesses in the community

- the reeds of the viliage as perceived by villagers themselves

- the population at greatest risk

- the health services presently availat?e and utilized

- viliagers' satisfaction with present health services

- villagers' receptivity toward the VHW cnncept

- villagers' perceptions of best 'VHW seiection and remuneration procecures

- the averaze villaçe nealth expenditure

- the willingness and capability of villagers to support? health vorker

- the special problens facing women and children in receiving care and information

- comunication thitworks within the village and between village and surrounding communities

- the avaiability of appropriate individuals in the villace to be trained as health workers

The findings of the Three province Survey presents much of this information; however. no attempt will be made here to review each item of information with implications for the establishment of a village hea?th worker progran. Instajd: cnly scue of the most saliant finsines will be discussed. 


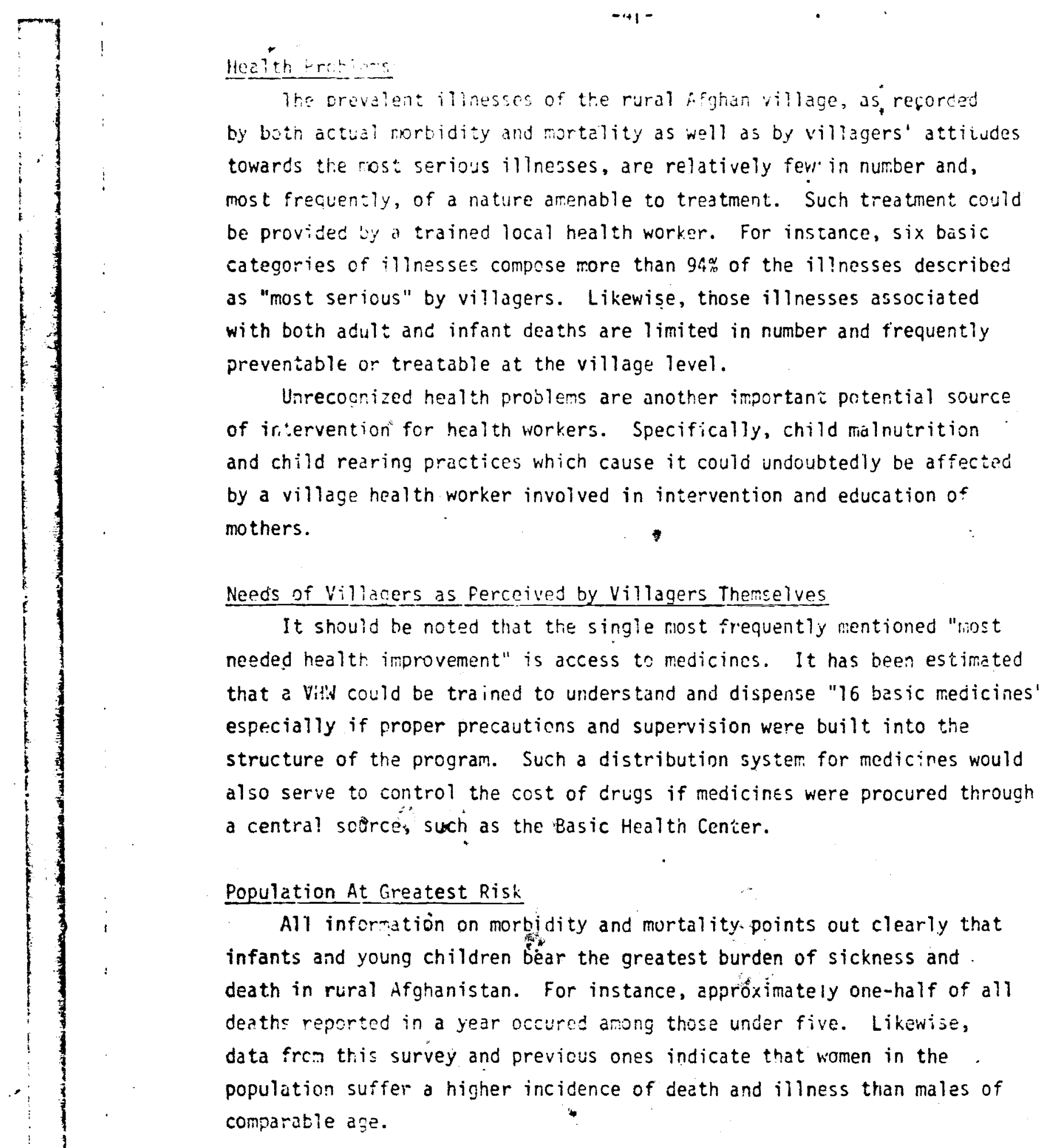


Services iresentiv Avariabie no the Villace

This survey, as do previous ones, points out vilioig that there is not a vacuum of hedith servicrs in the : ! ? jo. Thaic are many persons including midrives, phamacists, injectionists, shopkecpers, and others, who currently provide services and advice at the local ievel. While the quality of these services is not always viewed as satisfactory by respondents, thase providers do represent a cadre of local health workers who could be retrained and supervised to provide proper village health care. This is an especially important consideration in the case of midrives and other female health care providers, who might play an effective role in the education of village women.

Health Expenditures of Households

Respondents in this survey reported mear annual health expenditures of over 2,800 Afs. This amount is diviled among a wide variety of sources of care. In assessing whether a village could suppori a Vih--and many respondents report a willingness of the village to provide payment--it is important to consider the amount of money which a village currefitiy expends in its heaith seeking. As a theoretical estimate, it is userul to calculate the expenditures of an average size village of 300 households.

Village with 300 housenolds $\times 2800 \mathrm{Afs} /$ year $=840,000 \mathrm{~A} i \mathrm{~s} / \mathrm{yr}$.

If only $5 \%$ of this expenditure were used to support a VHW and supplies

3. "Hontfily village heaith worker budget $=48,000$ Afs/yr.

- Montily household allocation/month $=3,500$ Afs $=\quad 11.6 \mathrm{Afs}$

The theoretical nature of these estimates must be kept in mind. For instance, the mean: annual reported sperding less than 100 Afs for health care in the year prior to the survey. Nonetheless, the ev" ' nce does indicate that villages could become more self-sufficient if the appropriate means were available for the training and support of local health workers.

Feasibility of a VHll Pronram

The mojerity of respondents, 78: of males arid $95.2 \%$ of feriales, exprojsed the apinion that it would be possible to nave a village heaith 


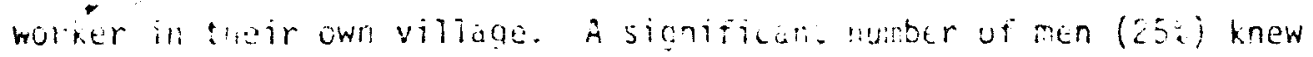
sonecas who they felt appropriats for the job. Ore linitation is the availability of village womer, who could be trained. Less than 30 of males and $10 \%$ of females felt thist it would be possible to recruit wohan from their village for a progran if it were to rafuire trainirg outside the viliage. Nonetheless, $20.3^{x}$ of men said that they would allicin their wives or daughters to participate in such a training program. The aggregate data indicates that, if proper consideration vere taken of cultural and village constraints, a Village Health horker program using either males or females could be set up.

Another constraint is literacy. Very few Afghan rural women are literate. Even though villaçers themselves place a high value on literacy as a prerequisite for a lill, one approach to circumventing this problem is to design training programs which do not presuppose or require literacy.

The interviewing experience also made abundantly clear that there are areas of rural Afghanistan whire a Vind progran would have only limited potential for success. For instance, in one pre-selected interviewing site the teams were met with resistance necessitating the selection of an alternitive sample site. The hostiitity which they experienced would appear to indicate that the villagers, for one reasor or another, do not eagerly await solutions or intrusions from the outside world. Such resistance is not the case for most villages, however, and where it does exist, it appears to be undiscuised, making a decision as to. whether a village is a good candidate for a VHW program relatively easy.

\section{Attitudes Towards Basic Heafth Centers}

The majority of househo?ds, both near the BHC and at a distance of up to $15 \mathrm{kms}$. away, reported having used its servicos. The attitudes of thọse who have used the BHC, while not unifomly complimentary, indicate a general satisfaction with the service it provides. This suggests that a plan to use BHC staff and facilities in the vital role of superisire, resupplying ard referral woild receive upport at the viliage ievel. 


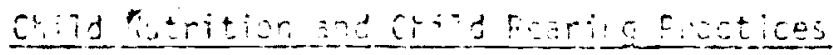

The resulss of tris survey-as weil as the frevious ond-opint out starkly the fact that malnutrition is one of the nost serious heaith probie-s--if int the met serious--iacing the Afghan villager. It is also increasingly covious that current child rearing attitudes and prastices are cirect cortributors to the infant and child malnutrition problem.

The implications or this for a VHW program is also clear. Any program for iraining of VHW's should provide comprehensive education in nutrition arici should stress the role of the worker in providing information and charging child feeding habits, including the timing of weanirg, length of breast feeding, types of foods for supplementation and the treatment and feeding of hildren with diarrhea.

The fact that women are severely limited in their ability to travel emphasizes the importance of recruiting and training female health workers for roles in health education and the provision of services in the village.

Again, this survey, in which over one-third of all children 1-4 years of age wero classified as malnourished by their am circumference measurement, strongly coints to a need for priority to be given to the anaiysis of national nutritional protiems and to the advisability of the formation of a National Nutrition Planring Commission to plan programs directed to these probiens.

\section{Comunicaticn}

While the findiros present cause for great concern in regard. to chilt health and leveis of knowledge among mothers, they also provide cause for optimism. For instante, the fact that over $50 \%$ of women report listening to the radic (62\% of these daily) is a hopeful sign.

Curreritly the radio is the most underused aid to development in Afghanistan. Prograns aimed at educating village vomen by radio appear to have potentia!.

Radio should also be mentioned as a means of constantly updating arid motivating Whis. The model for this can be found in the Radio forum used in Vigeria to compicate with village extension workers over a wide

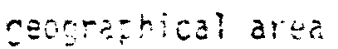


Wruther zesca for optinism is the large number of men (f9:) and women (3:) who are interestod in learning about means of spaciry intervais between births-ali improvement which has ramifications for both meternal and infant healsh. The impication of this firding for the roie of the viti in the provisica of family guidance information and services is clear. 